\title{
DISTRIBUCIÓN DE LA BECASINA GRANDE GALLINAGO STRICKLANDII (Gray, 1845) (SCOLOPACIDAE), EN CHILE.
}

\author{
DISTRIBUTION OF THE FUEGUIAN SNIPE GALLINAGO STRICKLANDII (Gray, 1845) \\ (SCOLOPACIDAE), IN CHILE.
}

La becasina grande (Gallinago stricklandii) es una de las tres especies del género Gallinago en Chile y una de las Scolopacidae menos conocida del país. Las pocas descripciones sobre su historia natural se basan en los apuntes de Reynolds (1935) quien describe vocalización, alimentación y el hábitat, en las islas del archipiélago del cabo de Hornos. También se registraron algunas características del tipo de hábitat en otras localidades de Chile austral (Philippi 1939). Poca atención ha recibido la descripción del plumaje, que junto a las pocas fotografías existentes hacen difícil su identificación en terreno. Algunos rasgos para su identificación, además del plumaje, forma del pico más recurvada en su porción distal y la diferencia de tamaño respecto a Gallinago paraguaiae, son el vuelo bajo y corto, a veces sin emitir vocalizaciones (Wijpkema \& Wijpkema 1999).

Los primeros registros para Chile se realizaron a partir de 1856 (MNHN) y posteriormente se reportó como parte de las colectas efectuadas por la expedición del HMS Alert en Magallanes (Sharpe 1881). Los primeros apuntes sobre su distribución en Chile indicaron que habita desde Valdivia $\left(39^{\circ}\right.$ S) y Chiloé hasta el extremo sur de Chile $\left(56^{\circ}\right.$ $\mathrm{S}$, Hellmayr 1932) y que existe migración poco regular (House 1945). Posteriormente Hellmayr
\& Conover (1948) mencionan nuevas localidades entre Valdivia y cabo de Hornos pero sin especificar fechas. Más tarde Goodall et al. (1951) extendieron el rango de distribución norte hasta Cautín ( $\left.37^{\circ} \mathrm{S}\right)$ y ocasionalmente en Concepción. A partir de la información recopilada hasta los años 60's (Philippi et al. 1956, Philippi 1964), trabajos subsecuentes repiten a los autores anteriores o entregan nueva información sin datos de apoyo. Aún menos es la información sobre la migración y reproducción, siendo los primeros registros de individuos juveniles y huevos provenientes desde la zona austral de Chile (Reynolds 1935).

La presente revisión aporta información sobre su distribución geográfica en base a especímenes de museo, registros de literatura y observaciones inéditas. Los datos inéditos corresponden a expediciones realizadas desde la región de Los Ríos hasta el cabo de Hornos, en los ambientes adecuados para localizar la especie según la literatura y registros casuales. El trabajo de campo incluyó desembarcos en diferentes islas de Fuego-Patagonia en los siguientes sectores y fechas: Punta Arenas a Puerto Williams en diciembre - abril, entre el 2001 y el 2003; Puerto Montt al cabo de Hornos en diciembre - febrero, entre los años 2002 al 2007; archipiélago Wollaston y cabo

\footnotetext{
${ }^{1}$ Feather Link, Inc. 1013 Westchester way, Cincinnati, OH 45244, USA.

${ }^{2}$ Casilla 19, Punta Arenas. alekusch@yahoo.com

${ }^{3}$ Los Angeles County Natural History Museum, Section of Ornithology, 900 Exposition Boulevard, Los Angeles CA 90007, USA. Dirección actual: Casilla 15, Melipilla, Chile. mma95@hotmail.com
} 
de Hornos en octubre del 2002, Puerto Montt en agosto del 2005, isla Noir en noviembre del 2003, 2004, 2005 y 2009; isla Hornos en agosto del 2006; varias localidades en el estrecho de Magallanes y zonas adyacentes en enero, febrero y marzo de 2007 y 2010; isla Wellington en diciembre y enero del 2006 y 2007.

En nuestros resultados entregamos 45 registros de G. stricklandii (Apéndice I), desde Ranquil $\left(36^{\circ} \mathrm{S}\right)$ hasta el cabo de Hornos (56 S), de los cuales 12 son inéditos. Durante el periodo estival se encontraron 32 y durante meses de invierno 13 registros.

\section{Periodo estival (septiembre - febrero)}

En septiembre se colectó un ejemplar en el lago Ranco ( $\left.40^{\circ} \mathrm{S}, \mathrm{AMNH}\right)$. En noviembre se han encontrado ejemplares en Tierra del Fuego $\left(53^{\circ} \mathrm{S}\right.$, Philippi et al. 1956), isla Noir (54\% S, Kusch et al. 2007) e isla Hornos (56 S, CZIP). Pocos encuentros se han realizado al norte de los $52^{\circ} \mathrm{S}$, por ejemplo en isla Asención (44\% S, Trimble 1943), archipiélago de los Chonos ( $\left.45^{\circ} \mathrm{S}, \mathrm{MNHN}\right)$, isla Wellington $\left(49^{\circ} \mathrm{S}\right.$, A. Kusch obs. pers.) y en isla Cisnes ( $52^{\circ} \mathrm{S}$, Philippi et al. 1956), los demás registros se centran en el archipiélago de las Wollaston y cabo de Hornos (Reynolds 1935, Olrog 1950, Venegas 1981).

Los datos reproductivos históricos dan cuenta de tres nidos, el 11 y 16 de diciembre en isla Hermite $e$ isla Wollaston y también otro en isla Barnevelt, todos con dos huevos (Reynolds 1935). También se encontró un polluelo en isla Hermite el 10 de enero de 1949 (Olrog 1950). Recientemente se encontró un adulto con dos polluelos de pocos días de nacidos el 17 de noviembre de 2006 en la zona sur occidental de la Isla Grande de Tierra del Fuego (D. Ascanio com. pers.).

A partir de 27 datos en periodo estival se pudo obtener información sobre el tipo de hábitat de los registros, donde en el $67,8 \%$ G. stricklandi se localizó en ambientes de turberas esfagnosas, $17,9 \%$ en bosques siempreverdes y $14,3 \%$ en vegas y pantanos. Debido a la dificultad para encontrar a esta especie, no es posible estimar su abundancia; sin embargo, el esfuerzo en terreno en los últimos 7 años, de aproximadamente $80 \mathrm{~km}$ recorridos en isla Wellington, isla Noir, península de Brunswick e isla Riesco solo dieron cuenta de 5 ejemplares. Tan solo en la península de Brunswick e isla Riesco $\left(53^{\circ} \mathrm{S}\right)$ se recorrieron ca. $30 \mathrm{~km}$ durante enero del 2010 y se encontró un solo ejemplar.

\section{Periodo invernal (marzo - agosto)}

En marzo y la primera semana de junio existieron observaciones en la zona austral $\left(47^{\circ} \mathrm{S}-55^{\circ} \mathrm{S}\right.$, Sharpe 1881, Trimble 1943, Imberti 2005). Hacia fines de junio, en julio, agosto y principios de septiembre, los registros fueron entre Ranquil (ca. $37^{\circ}$ S) hasta Maullin (ca. $42^{\circ} \mathrm{S}$ ) en base a especímenes de museo y observaciones personales (Apéndice I). Varios autores mencionan que no se ha comprobado el estatus migratorio de la especie en la zona sur de Chile (Hellmayr 1932, House 1945, Goddall et al. 1951). El avistamiento en isla Hornos en junio del 2006 (T. Ibarra com. pers.) y en Puerto Bueno (51 $\mathrm{S}$, Imberti 2005) podrían indicar que durante el invierno las poblaciones australes no migran totalmente hacia latitudes más bajas de Chile, o bien son evidencia de migraciones intrarregionales (Venegas \& Jory 1979).

La becasina grande es una especie que posee un rango de distribución estival restringido a la zona archipelágica al sur de los $48^{\circ} \mathrm{S}$, y durante invierno principalmente la zona costera entre los $36^{\circ} \mathrm{S}$ y $48^{\circ} \mathrm{S}$. Más del $90 \%$ del territorio estival disponible para la becasina grande se encuentra protegido por el Sistema Nacional de Áreas Silvestres Protegidas del Estado (SNASPE); sin embargo, durante los últimos 30 años su rango de distribución invernal ha sido transformado a extensas zonas de desarrollo silvoagropecuario (Pliscoff et al. 20051, Pauchard et al. 2006, Aguayo et al. 2009). Aunque la becasina grande posee estado de conservación a nivel nacional de Vulnerable (Glade 1993), no existe diferenciación en zonas del territorio nacional con diferentes usos de suelo o protección. Debido a la pérdida de hábitat y su baja frecuencia de registros, se recomienda seguir el criterio de zonas geográficas y categorías de conservación del Servicio Agrícola y Ganadero, otorgándole para la zona austral de Chile con la categoría de Rara y para la zona sur como Vulnerable. 


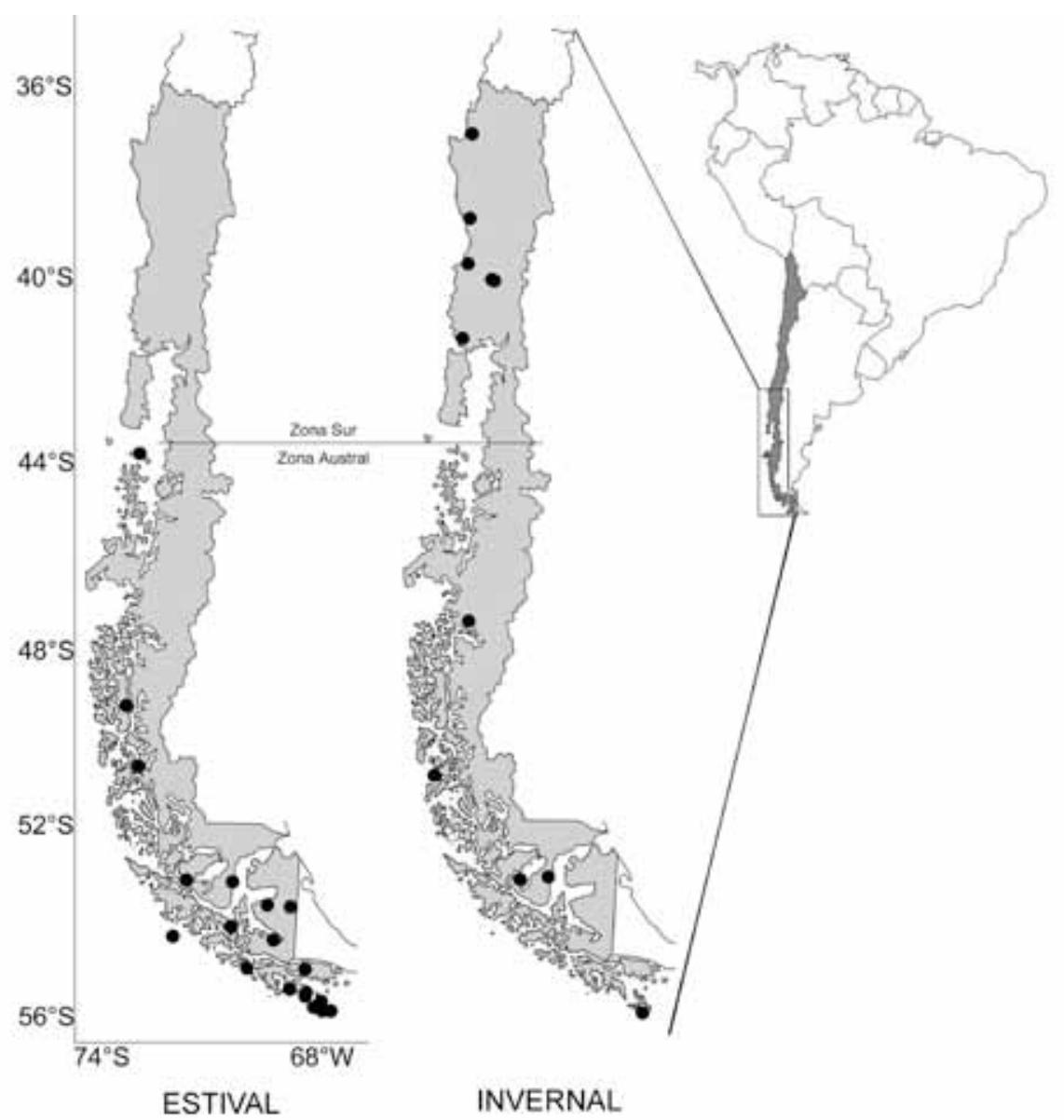

Fig. 1. Distribución de registros de Gallinago stricklandii en Chile. El periodo estival se considera desde de septiembre hasta febrero y el periodo invernal entre marzo y agosto.

\begin{tabular}{clcc}
\hline Fecha & \multicolumn{1}{c}{ Localidad } & Lat. $\left.{ }^{\circ} \mathrm{S}\right)-$ Lon. $\left(^{\circ} \mathrm{W}\right)$ & Fuente \\
\hline 11-mar-1939 & Pto. Huemul, Aisén & $47^{\circ} 38^{\prime}-73^{\circ} 42^{\prime}$ & Trimble 1943 \\
14-mar-1880 & Swallow Bay, Magallanes & $53^{\circ} 30^{\prime}-72^{\circ} 45^{\prime}$ & Sharpe 1881 \\
xx-mar-2001 & Pta. Arenas, Magallanes & $53^{\circ} 00^{\prime}-70^{\circ} 55^{\prime}$ & CZIP \\
17-abr-2003 & Isla Noir, Magallanes & $54^{\circ} 28^{\prime}-73^{\circ} 02^{\prime}$ & R. Matus, obs. pers. \\
xx-abril-1998 & Pta. Arenas, Magallanes & $53^{\circ} 00^{\prime}-70^{\circ} 55^{\prime}$ & R. Matus, obs. pers. \\
02-Jun-2006 & Is. Hornos, Magallanes & $55^{\circ} 57^{\prime}-65^{\circ} 13^{\prime}$ & T. Ibarra, obs. pers \\
07-Jun-2003 & Puerto Bueno, Magallanes & $50^{\circ} 59^{\prime}-74^{\circ} 13^{\prime}$ & Imberti 2003 \\
29-jun-1946 & Ranquil, Concepción & $36^{\circ} 38^{\prime}-72^{\circ} 34^{\prime}$ & FB \\
xx-jul-1941 & Lago Ranco, Los Lagos & $40^{\circ} 21^{\prime}-72^{\circ} 40^{\prime}$ & RAPH/AMNH \\
xx-jul-1942 & Is. de Pichibureo, Los Lagos & $40^{\circ} 16^{\prime}-72^{\circ} 32^{\prime}$ & YPM \\
03-ago-2005 & Maullín, Los Lagos & $41^{\circ} 38^{\prime}-73^{\circ} 37^{\prime}$ & A. Kusch, obs. pers. \\
xx-ago-1856 & Valdivia, Los Ríos & $39^{\circ} 48^{\prime}-73^{\circ} 14^{\prime}$ & MNHN \\
xx-ago-1938 & Toltén, Cautín & $39^{\circ} 13^{\prime}-73^{\circ} 13^{\prime}$ & YPM
\end{tabular}




\begin{tabular}{|c|c|c|c|}
\hline 01-sep-1939 & Lago Ranco, Los Lagos & $40^{\circ} 21^{\prime}-72^{\circ} 40^{\prime}$ & AMNH \\
\hline 15-nov-2003 & Is. Noir, Magallanes & $54^{\circ} 28^{\prime}-73^{\circ} 02^{\prime}$ & M. Marín, obs. pers. \\
\hline 17-nov-2006 & Seno Chico, Magallanes & $54^{\circ} 25^{\prime}-71^{\circ} 07^{\prime}$ & D. Ascanio, obs. pers. \\
\hline 18-nov-1981 & Isla Hornos, Magallanes & $55^{\circ} 57^{\prime}-65^{\circ} 13^{\prime}$ & CZIP \\
\hline 19-nov-2004 & Is. Noir, Magallanes & $54^{\circ} 28^{\prime}-73^{\circ} 02^{\prime}$ & M.M., A. K., obs. pers. \\
\hline xx-nov-1952 & Ea. Cameron, Magallanes & $53^{\circ} 38^{\prime}-69^{\circ} 39^{\prime}$ & Philippi et al. 1956 \\
\hline 08-dic-1988 & Is. Hornos, Magallanes & $55^{\circ} 57^{\prime}-65^{\circ} 13^{\prime}$ & Wijpkema \& Wijpkema 1999 \\
\hline 09-dic-1941 & Isla Caroline, Magallanes & $55^{\circ} 25^{\prime}-69^{\circ} 32^{\prime}$ & $\mathrm{AMNH}$ \\
\hline 11-dic-1932 & Isla Guffern, Magallanes & $55^{\circ} 26^{\prime}-68^{\circ} 04^{\prime}$ & Reynolds 1935 \\
\hline 11-dic-1932 & Isla Packsaddle, Magallanes & $52^{\circ} 54^{\prime}-68^{\circ} 05^{\prime}$ & Reynolds 1935 \\
\hline 16-dic-1932 & Isla Barnevelt, Magallanes & $55^{\circ} 43^{\prime}-67^{\circ} 23^{\prime}$ & Reynolds 1935 \\
\hline 30-dic-1948 & Río Douglas, Magallanes & $55^{\circ} 10^{\prime}-68^{\circ} 07^{\prime}$ & Olrog, 1950 \\
\hline 17-dic-2007 & Is. Vauverlandt, Magallanes & $55^{\circ} 20^{\prime}-67^{\circ} 59^{\prime}$ & M. Marín, obs. pers. \\
\hline 31-dic-1914 & Is. Wollaston, Magallanes & $55^{\circ} 43^{\prime}-67^{\circ} 23^{\prime}$ & $\mathrm{AMNH}$ \\
\hline xx-dic-1945 & Is. Cisnes, Magallanes & $51^{\circ} 46^{\prime}-72^{\circ} 32^{\prime}$ & Philippi et al. 1956 \\
\hline$x x-\operatorname{dic}-x x x x$ & $\begin{array}{l}\text { Cabo de Hornos, } \\
\text { Magallanes }\end{array}$ & $55^{\circ} 57^{\prime}-65^{\circ} 13^{\prime}$ & Reynolds 1935 \\
\hline$x x-d i c-x x x$ & San Sebastián, Magallanes & $53^{\circ} 20^{\prime}-68^{\circ} 40^{\prime}$ & Humprey et al.1970 \\
\hline 02-ene-1949 & Is. Amarilla, Magallanes & $55^{\circ} 33^{\prime}-68^{\circ} 00^{\prime}$ & Olrog 1950 \\
\hline 02-ene-1949 & Is. Grevy, Magallanes & $55^{\circ} 34^{\prime}-67^{\circ} 38^{\prime}$ & Olrog 1950 \\
\hline 03-ene-1915 & Is. Wollaston, Magallanes & $55^{\circ} 43^{\prime}-67^{\circ} 23^{\prime}$ & AMNH \\
\hline 05-ene-2006 & Is. Hornos, Magallanes & $55^{\circ} 57^{\prime}-65^{\circ} 13^{\prime}$ & M. Marín, obs. pers. \\
\hline 10-ene-1949 & Is. Hermite, Magallanes & $55^{\circ} 52^{\prime}-67^{\circ} 20^{\prime}$ & Olrog 1950 \\
\hline 12-ene-1939 & Is. Herschel, Magallanes & $55^{\circ} 51^{\prime}-67^{\circ} 17^{\prime}$ & Olrog 1950 \\
\hline 13-ene-1915 & Is. Londonderry, Magallanes & $55^{\circ} 03^{\prime}-70^{\circ} 35^{\prime}$ & AMNH \\
\hline 15-ene-2010 & Estero Cóndor, Magallanes & $53^{\circ} 19^{\prime}-72^{\circ} 41^{\prime}$ & A. Kusch, obs. pers. \\
\hline 18-ene-2008 & Pe. Broome, Magallanes & $49^{\circ} 22^{\prime}-74^{\circ} 25^{\prime}$ & A. Kusch, obs. pers. \\
\hline 31-ene-1923 & Is. Ascención, Los Lagos & $43^{\circ} 53^{\prime}-73^{\circ} 50^{\prime}$ & Trimble 1943 \\
\hline 31-ene-2009 & $\begin{array}{l}\text { Fiordo Marinelli, } \\
\text { T. del Fuego }\end{array}$ & $54^{\circ} 24^{\prime}-69^{\circ} 37^{\prime}$ & C. Silva, obs. pers. \\
\hline xx-ene-1858 & Ar. de los Chonos & $45^{\circ} 00^{\prime}-74^{\circ} 00^{\prime}$ & MNHN \\
\hline 20-feb-1980 & Isla Wollaston, Magallanes & $55^{\circ} 43^{\prime}-67^{\circ} 23^{\prime}$ & Venegas 1981 \\
\hline 20-feb-1980 & Isla Bayly, Magallanes & $55^{\circ} 40^{\prime}-67^{\circ} 40^{\prime}$ & Venegas 1981 \\
\hline$x x-x x x-1839$ & Ba. Orange, Magallanes & $55^{\circ} 31^{\prime}-68^{\circ} 03^{\prime}$ & USNM \\
\hline
\end{tabular}

\section{AGRADECIMIENTOS}

Agradecemos a las personas encargadas de las colecciones de museo que entregaron datos de los especímenes de Becasina grande: Claudio Venegas, Instituto de la Patagonia (CZIP); Gary Graves y James Dean, United States National Museum (USNM); Kristoff Zyskowski, Yale Peabody Museum (YPM); Sra. Erica de Behn; Colección "Rudolfo Armando Philippi" (RAPH); Mark Adams, Natural History Museum (Tring, NHM); Juan Carlos Torres-Mura, Museo Nacional de Historia
Natural (MNHN); American Museum of Natural History (AMNH). Agradecemos especialmente a Ricardo Matus por enriquecer el manuscrito y entregar sus registros, también a Tomás Ibarra y Daniel Ascaino por sus observaciones. A Fantástico Sur Birding, la tripulación del M/V Terra Australis, M/V Mare Australis, M/S Nordnorge, LAM Austral II, yate Chonos, LSG Hallef, Harley Benavente y Centro EULA por el transporte hacia diferentes localidades del sur de Chile. 
LITERATURA CITADA

Aguayo, M., A. Pauchard, G. Azócar \& O. Parra 2009. Cambio del uso del suelo en el centro sur de Chile a fines del siglo XX. Entendiendo la dinámica espacial y temporal del paisaje. Revista Chilena de Historia Natural 82: $361-374$.

Glade, A. 1993. (Ed.) Libro rojo de los vertebrados terrestres de Chile. Corporación Nacional Forestal, Santiago, Chile. $2^{a}$ edición, 68 pp.

Goodall, J. D., A.W.Johnson \& R.A. Philippi 1951. Las aves de Chile. Su conocimiento y sus costumbres. Vol. 2. Platt Estab. Gráficos, Buenos Aires.

Hellmayr, C. E. 1932. The birds of Chile. Zool. Series, Pub. 308, Volume XIX, Field Mus. Nat. Hist., Chicago, Illinois.

Hellmayr, C. E. \& B. Conover 1948. Catalogue of birds of the Americas and the adjacent islands. Zool. Series Field Mus. Nat. Hist. Vol 13, part 1, number 3.

Housse, R. 1945. Las aves de Chile en su clasificación moderna su vida y costumbres. Ediciones de la Universidad de Chile, Santiago.

Humphrey, P. S., D. Bridge, P. W. Reynolds \& R. T. Peterson 1970. Birds of Isla Grande (Tierra del Fuego). Smithsonian Institution and University of Kansas Mus. Nat. History, Lawrence, Kansas.

Imberti, S. 2005. Distribución otoñal de aves marinas y terrestres en los canales chilenos. Anales Instituto Patagonia (Chile) 33: 21 - 30.

Jaramillo, A. 2003. Birds of Chile. Princeton Field Guides. Princeton University Press.

Kusch, A., M. Marín, D. Oheler \& S. Drieschman 2007. Notas sobre la avifauna de isla Noir (54 $\left.28^{\circ} \mathrm{S}-73^{\circ} 00^{\prime} \mathrm{W}\right)$. Anales Instituto Patagonia (Chile) 35: 61 - 66.

Olrog, C. C. 1950. Mamíferos y aves del archipiélago de Cabo de Hornos. Acta Zool. Lilloana 9: $505-532$.

Pauchard, A., M. Aguayo, E. Peña \& R. Urrutia 2006. Multiple effects of urbanization on the biodiversity of developing countries: the case of a fast-growing metropolitan area (Concepción, Chile). Biological Conservation 127: $272-281$.

Paynter, R. A. Jr. 1988. Ornithological Gazetter of Chile. Museum of Comparative Zoology, Hardvard University, Cambridge, Massachussets.

Philippi, R.A. 1939. Contribución al conocimiento de la ornitología de la provincia de Aysén (Chile). Revista Chilena de Historia Natural 42:4-20

Philippi, R.A. 1964. Catálogo de las aves de Chile con su distribución geográfica. Inv. Zool. Chilenas 11:1-179.

Philippi, R.A., A.W. Johnson, J.D. Goodall \& F. Behn 1956. Notas sobre aves de Magallanes y Tierra del Fuego. Bol. Mus. Hist. Nat. (Segunda Parte) 26:1-57.

Reynolds, P.W. 1935. Notes on the birds of Cape Horn. Ibis 65-101.

Sharpe, R. B. 1881. Account of the zoological collections made during the survey of the H.M.S. "Alert" in the Straits of Magellan and on the coast of Patagonia. Proceedings of the Zoological Society of London 1881: 1 - 14 .

Trimble, R. 1943. Birds collected during two cruises of the "Vagabondia" to the best coast of South America. Annals of the Carnegie Museum Vol. 29: 409 - 441.

Venegas, C. 1981. Aves de las islas Wollaston y Bayly, Archipiélago del Cabo de Hornos. Anales Instituto Patagonia (Chile) 12: 213 - 219.

Venegas, C. \& J. Jory 1979. Guía de Campo para las aves de Magallanes. Publicaciones Instituto de la Patagonia, Serie Monografías $\mathrm{N}^{\circ}$ 11, Punta Arenas.

Venegas, C. \& W. Sielfeld 1998. Catálogo de los vertebrados de la región de Magallanes y Antártica Chilena. Ed. Universidad de Magallanes.

Wijpkema, J. \& T. Wijpkema 1999. Fuegian Snipe. Dutch Birding 21: 205 - 206. APÉNDICE I. Lista de registros de Gallinago stricklandii en Chile. 